\title{
IDENTIFICATION OF X-RAY SOURCES AT CERRO TOLOLO INTER-AMERICAN OBSERVATORY
}

\author{
W. E. KUNKEL and V. M. BLANCO
}

The telescope used for identification of X-ray sources at the Cerro Tololo InterAmerican Observatory is the Curtis Schmidt Telescope. This telescope is located at Tololo by agreement with the University of Michigan. The field of the telescope is $5^{\circ} \times 5^{\circ}$ and its correcting aspheric plate has negligible chromatic aberration over the range 3200 to $10000 \AA$. This latter feature makes it an ideal instrument for multicolor survey work.

For optical identification of X-ray sources, the technique followed aims at the discovery of objects with strong ultraviolet excess. Eastman 103aO plates are used, and a given plate is exposed first through a Schott UG1 or UG2 filter, and then, after the telescope is moved about $30^{\prime \prime}$, exposed again through a GG13 filter.

Experience shows that relative exposures of about 6 to 1 for the ultraviolet exposure as compared with the blue exposure, yield a pair of images that are comparable in size and density for earlier O-type stars. Objects whose ultraviolet image, in these plates, is stronger than the blue image are likely to have strong ultraviolet excess. To reach magnitude 18 , exposures of $7 \mathrm{~min}$ for the blue and $42 \mathrm{~min}$ for the ultraviolet are required. To this limit, experience shows that there is an expectancy, depending on location in the Milky Way, of from one to four objects with strong ultraviolet excess per 4 square degrees in the neighborhood of the galactic center. Thus it is rather important that the smallest possible error box is available before an attempt at identification is made.

To follow up tentative candidate objects (1) objective spectra of about $500 \AA / \mathrm{mm}$ dispersion at $\mathrm{H} \gamma$ are obtained, yielding information to about magnitude $\mathrm{B}=15$. The principal telescope for follow-up photometry and spectrography is a 60 -inch $(1.5 \mathrm{~m})$ reflector with modified Ritchie-Chretien optics. A Cassegrain image-tube spectrograph can be used with this telescope, yielding usable spectra to $B=17$.

Identification at Cerro Tololo has been attempted for about 12 sources and tentative candidates have been obtained for Cen XR-2, GX3 +1, and Vela X-1.

Work on the first of these, Cen XR-2, was done late in 1967 and the central star in the irregular planetary nebula NGC 5189 was picked out as a possibility (Blanco, 1967). About the time that this identification survey was going on, Feast in South Africa also mentioned this planetary nebula as a possibility (Feast, 1967). Photometry of this star confirmed its marked ultraviolet color.

The main spectral features are exceedingly strong and broad emission of OVI 3811 and 3834 and of HeII 4686. The spectrum is thus not at all like that of Sco XR-1. Prior to this work, this type of spectrum had been found by other investigators in a number of central stars in planetary nebulae. A sequence can indeed be established of a number of these objects, and the star in NGC 5189 appears to be, because of the 
strength of the OVI 3811 and 3834 emissions, an extreme example of the members of such a sequence.

The identification of Cen XR-2 with the star responsible for the excitation of the planetary nebulae NGC 5189 is no longer fashionable. The peculiar variable WX Cen, which shows spectral and variability features that are similar to the object identified as Sco XR-1, is currently a candidate favored by several investigators (Eggen and Lyngå, 1968).

Nevertheless, it is worth pointing out that another tentative identification made at Tololo for the source GX3 +1 resulted in an object having photometric and spectroscopic features that are practically identical with those of the star in NGC 5189 (Blanco et al., 1968).

In the case of $\mathrm{GX} 3+1$, the error box was about $15 \mathrm{sec}$ of arc on a side, and about $\frac{1}{16}$ of a square degree in area.

The ultraviolet object was found within the box. From frequency of ultraviolet objects in the surroundings of GX3 +1 , the expectancy of one such object in the kind of survey made, is less than one in fifty. This low expectancy is the main argument in favor of the identification of $\mathrm{GX} 3+1$, and in turn, the fact that this candidate and the central star in NGC 5189 have similar colors and spectral features gives support to the latter identification.

A search for an optical candidate for the X-ray source in Vela (Vela XR-1, or GX263+3) was conducted in a region of about ten square degrees centered on $8^{\mathrm{h}} 57^{\mathrm{m}}$ and $-41^{\circ} 15^{\prime}$ (Epoch 1950) using the double exposure method previously described. Ten candidates found this way, all fainter than $B=15.3$, were examined photoelectrically with the 60 -inch reflector. The hottest (or most ultraviolet) object at

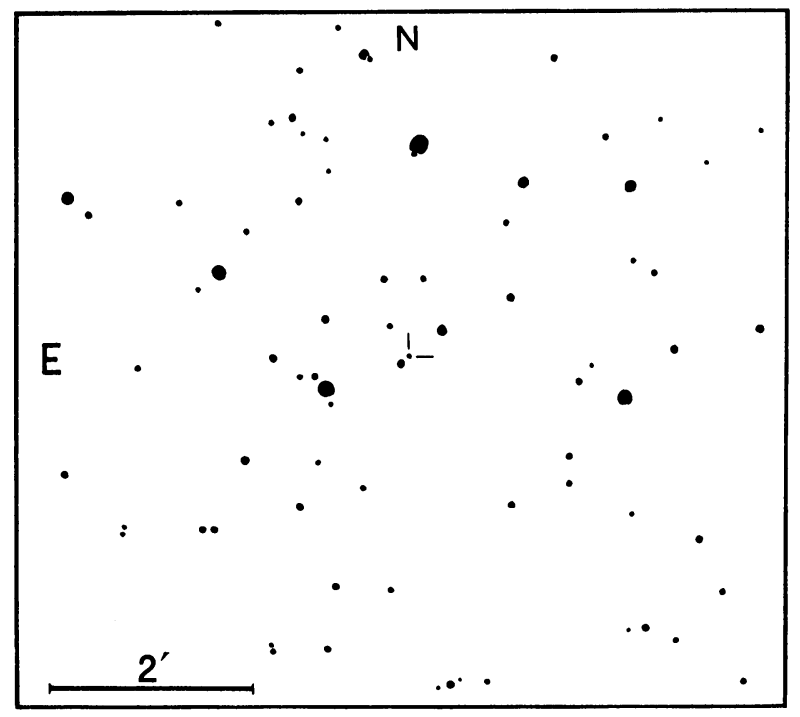

Fig. 1. Identification Chart for the Optical Candidate of Vela XR-1. 30-minute exposure on 103a-E with RG-2 filter, Schmidt telescope. 
position (Epoch 1950) $\alpha=8^{\mathrm{h}} 57^{\mathrm{m}} 55^{\mathrm{s}}$ and $\delta=-41^{\circ} 13^{\prime} .9$ is quite close to the center of the uncertainty box published by Gursky et al. (1968), well within its borders. A chart for identification is shown in Figure 1. The magnitude and colors, determined from a single night's observations, are $B=17.3 \pm 0.1, B-V=+0.21 \pm 0.10$, and $\mathrm{U}-\mathrm{B}=-0.92 \pm 0.12$. The rather large mean errors result from a close optical companion about five seconds of arc distant, near p.a. $\simeq 135^{\circ}$. Photometry for this star is $B=15.92 \pm 0.07, B-V=+0.91 \pm 0.05$, and $U-B=+0.07 \pm 0.08$. Since some trouble was encountered with the equipment, these values should be considered provisional. Within these limits, the colors lie near the black body line for both stars, as though both were white dwarfs. Unwidened image-tube spectra at $100 \AA / \mathrm{mm}$ dispersion of the fainter, hotter star show a smooth continuum with no lines in either emission or absorption, and in one of two spectra of the brighter, cooler star, $\mathrm{H}$ and $\mathrm{K}$ of CaII show faintly in absorption.

Since a number of conflicting interpretations of these preliminary results appear possible, further observations of this pair of stars are planned for the next season.

\section{References}

Blanco, V. M.: 1967, I.A.U. Circular No. 2035.

Blanco, V. M., Kunkel, W. E., and Hiltner, W. A.: 1968, Astrophys. J. 152, L137. See also Freeman, K. C., Rodgers, A. W., and Lyngå, G.: 1968, Nature 219, 251.

Eggen, O. J. and Lyngå, G.: 1968, Astrophys. J. 153, L195.

Feast, M. W.: 1967, Nature 215, 1158.

Gursky, H., Kellogg, E. M., and Gorenstein, P.: 1968, Astrophys. J. 154, L71. 